$\stackrel{N}{N}$

Global Journals Inc

is

\title{
Machine Reliability Optimization by Genetic Algorithm Approach
}

By Ngnassi Djami Aslain Brisco, Nzie Wolfgang \& Doka Yamigno Serge

Abstract- To define the reliability network of a system (machine), we start with a set of components arranged in an appropriate topology (series, parallel, or parallel-series), choose the best terms of the ratio performance / cost, and gather by links with the aim to combine them. This process requires a long time and effort, given the very large number of possible combinations, which becomes tedious for the analyst. For this reason, it is essential to use an appropriate optimization approach when designing any product. However, before trying to optimize, it is necessary to have a reliability assessment method. The objective of this paper is to display a meta-heuristic method, which is sustained on the genetic algorithm (GA) to improve the machines reliability. To achieve this objective, a methodology that consists of presenting the functionalities of genetic algorithms is developed. The result achieved is the proposal of a reliability network for the optimal solution.

Keywords: reliability, cost, reliability network, topology.

GJRE-A Classification: FOR Code: 091399

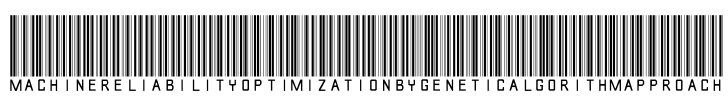

Strictly as per the compliance and regulations of:

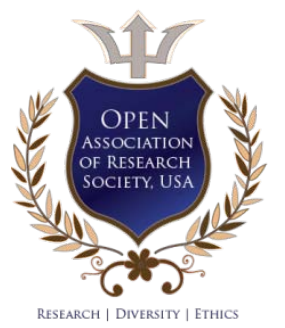

(C) 2020. Ngnassi Djami Aslain Brisco, Nzie Wolfgang \& Doka Yamigno Serge. This is a research/review paper, distributed under the terms of the Creative Commons Attribution-Noncommercial 3.0 Unported License http://creativecommons.org/licenses/by$\mathrm{nc} / 3.0 /$ ), permitting all non commercial use, distribution, and reproduction in any medium, provided the original work is properly cited. 


\title{
Machine Reliability Optimization by Genetic Algorithm Approach
}

\author{
Ngnassi Djami Aslain Brisco ${ }^{\alpha}$, Nzie Wolfgang ${ }^{\sigma} \&$ Doka Yamigno Serge ${ }^{\rho}$
}

Abstract- To define the reliability network of a system (machine), we start with a set of components arranged in an appropriate topology (series, parallel, or parallel-series), choose the best terms of the ratio performance / cost, and gather by links with the aim to combine them. This process requires a long time and effort, given the very large number of possible combinations, which becomes tedious for the analyst. For this reason, it is essential to use an appropriate optimization approach when designing any product. However, before trying to optimize, it is necessary to have a reliability assessment method. The objective of this paper is to display a meta-heuristic method, which is sustained on the genetic algorithm (GA) to improve the machines reliability. To achieve this objective, a methodology that consists of presenting the functionalities of genetic algorithms is developed. The result achieved is the proposal of a reliability network for the optimal solution.

Keywords: reliability, cost, reliability network, topology.

\section{InTRODUCTION}

T he fundamental function of a system is to provide its customers with a fairly economical cost, acceptable reliability required. These constraints require an optimal design. In the engineering context, the fundamental interest of manufacturers is to find a balance between the reliability of a system and its cost. These two factors constitute the most important decision variable for optimizing a system. This is generally manifested in minimizing the cost under the constraint of reliability on the one hand, and in improving performance to meet the needs of customers under the constraint of cost on the other.

In this area, researchers have developed and improved many methods and algorithms. The set of methods can be divided into two main categories: exact methods, which guarantee to obtain an optimal solution for problems of reasonable size, and approximate methods (heuristics and meta-heuristics), which give good solutions. Quality, without guarantee of optimality, but for the benefit of shorter calculation time.

Author a: Department of Fundamental Sciences and Techniques of Engineer, EGCIM, University of Ngaoundere, Cameroon.

e-mail:ngnassbris@yahoo.fr

Author $\sigma$ : Department of Mechanical Engineering, ENSAl, University of Ngaoundere, Cameroon.

Author $\rho$ : Department of Physics, Faculty of Sciences, University of Ngaoundere, Cameroon.
If the exact methods are based on the enumeration, often implicitly, for the search for the set of solutions of the search space, then the approached methods rather require random processes in the exploration of potential solutions, and this to deal with the combinatorial explosion generated when using the exact methods. In this perspective, we are essentially seeking in this work to integrate an efficient and adapted optimization method to solve the problem of optimizing reliability by taking into account the most relevant constraints.

Given the large number of configurations that can result from a set of components, the procedure of enumerating all the possible architectures is no longer pleasant. Consequently, it is necessary to opt for an approximate method, which will make it possible to find the solution closest to the optimal solution because it is not obvious to examine all the possibilities.

In this perspective, we opted for the method of genetic algorithms as an optimization technique. It is considered an effective meta-heuristic method in the field of dependability. It is inspired by genetic biology and is based on the principle of the search for evolution. It does not guarantee an exact solution, but it generates a solution close to the optimum (Painton and Campbell [1], Levitin and Lisnianski [2]).

\section{il. The Functioning of the Genetic Algorithm}

\section{a) Origin and principle}

Genetic algorithms (GA) are heuristic optimization algorithms based on the principles of natural selection and genetics. The researcher Rechenberg [3], is the first scientist who introduced evolutionary algorithms by publishing his work "Evolution strategies." These algorithms are broadly inspired by Darwin's theory of evolution published in 1859. Next, Holland [4] proposed the first genetic algorithms to solve combinatorial optimization problems, and they were also developed by the work of David Goldberg published in 1989 (Goldberg [5] and Goldberg [6]).

The aim of the genetic algorithm is to bring up, from one generation to another, the candidates (potential solutions) most suited to solving the problem. Each generation is made up of a defined number of individuals, these form a population, and each of them represents a point in the search space. Each individual (chromosome) has information coded in the form of a 
chain of characters that analogically constitutes genes. Then the passage from one generation to another is carried out based on the process of evolution by the use of evolutionary operators like selection, crossing, and mutation.

Their operating principle is quite simple. From an initial population created at random, composed of a set of individuals (chromosomes), we proceed to the evaluation of their parent qualifications to highlight the best suited, as long as the least effective are rejected. Then, the most qualified individuals are chosen by privileged selection by giving them a chance to reproduce by crossing and mutating via the two operators of crossing and mutation. Then by relaunching this process several times, the optimal solution can be refined by passing from one generation to another (Douiri et al. [7]).

b) Description of the formalism used

Clustering is a process that partitions a set of data into meaningful subclasses (clusters or clusters).

The convergence of genetic algorithms has been demonstrated for many problems, although optimality cannot be guaranteed. The ability of a genetic approach to find the right solution often depends on the adequacy of the coding, the evolution operators, and the measures of adaptation to the problem being addressed. The method proposed here is based on genetic algorithms (Goldberg [6]) and evolutionary strategies (Schewefel [8]). It combines the principle of survival of the ablest individuals and genetic combinations for an elitist research mechanism. The genetic method produces new solutions (children) by combining existing solutions (parents) selected from the population, or by mutation. The central idea is that parent solutions will tend to produce superior child solutions in terms of adaptation so that ultimately a solution obtained is optimal.

In this study, we used a genetic method previously defined by Bicking et al. [10] with a definition of the chromosome and the operators of selection, combination, and mutation concerned. Unlike genetic algorithms, the genetic method used is designed to minimize and not maximize. This method, like genetic algorithms, is not limited by assumptions about the objective function and research space, such as continuity or differentiability. It uses a population of points simultaneously by contrast with usual methods using only one point. Genetic operators are elitistically improving the search process to find the global optimum. There are more complicated genetic operators, but the basic operators and their various modifications can generally be applied. The choice of these operators depends on the nature of the problem and the performance requirements. The genetic algorithm that we are going to implement is as follows, where the process is applied to iteration $k$ :
1. Data coding;

2. Generation of the initial population $P_{0}$ of $N$ individuals;

3. Assessment of the adaptation of all individuals in the population;

4. Selection of a proportion of the best individuals (parents for the production of new individuals);

5. The Crossing of all individuals in the population $P_{k}$ two by two with a probability $P_{m}$, we will have $N$ children noted $C_{k}$;

6. Mutation of all individuals in the population, we will have $N$ elements noted $M_{k}$;

7. Choice of the most suitable individuals, i.e., those who optimize the objective function;

8. If the stop test is verified, stop, otherwise return to step $a$.

We will choose, as a stop test in our implementation, a finite number of iterations.

It is important to note that the stopping criterion can be several cycles of the algorithm (number of generations), the average of the adaptations of individuals, a convergence factor, etc.

An individual represents a vector of decision variable (parameters), and its adaptation is measured by the objective function. The formalism and the genetic operators are detailed below.

\section{i. Data coding}

The first step is to properly define and code the problem. That step associates with each point of the search space a specific data structure called a chromosome, which will characterize each individual in the population. This step is considered to be the most important step in GA because the success of these algorithms depends heavily on how individuals are coded.

There are different choices for coding a chromosome, this choice being a very important factor in the progress of the algorithm so it must be well suited to the problem being addressed:

- Binary coding: It is the most used coding. The chromosome is coded by a string of bits (which can take the value 0 or 1) containing all the information necessary to describe a point in space;

- Multi-character coding: this is often more natural. We are talking about multiple characters as opposed to bits. A chromosome is then represented by a series of numbers or characters, each representing a gene;

- Coding in the form of a tree: this coding in tree structure starts from a root (comprising several parts equal to the number of initial individuals), from which one or more children can be derived. The tree then builds up gradually, adding branches to each new generation. 


\section{ii. Generation of the initial population}

Each chromosome is the potential result of the optimization problem. We define a chromosome as a chain composed of genes, which are the parameters (decision variables) to find. The value of a gene is called an allele. The possible value of an allele is an integer or a real value. Each gene is created randomly, using equation 1

$$
a_{j}=\left(a_{j}\right)_{l}+\left(\left(a_{j}\right)_{u}-\left(a_{j}\right)_{l}\right) \times \gamma_{j}
$$

Where:

- $\gamma_{j} \in\{0 ; 1\}$ is chosen randomly

- $\left(a_{j}\right)_{l},\left(a_{j}\right)_{u}$ are the minimum and maximum limits of the allele $a_{j}$. They are chosen according to the problem to be treated.

Each chromosome, called an individual in a haploid representation, can be written:

$$
X_{i}=\left[a_{1}, \ldots, a_{j}, \ldots, a_{m}\right]
$$

With:

- $\quad m$ is the number of genes

- $i=1, \ldots, N$ and $N$ is the size of the population (number of individuals).

All the constraints are taken into account in the initial phase of population creation. When an individual is created, if the constraints are respected, this individual is integrated into the initial population; otherwise, it is not. At the start of the algorithm, the initial population contains individuals.

The length of the chromosome $\mathrm{m}$ and the size of the population $\mathrm{N}$ is two of the four adjustment parameters of the genetic method.

\section{iii. Objective function and adaptation}

We evaluate the different solutions proposed to treat them according to their relevance and to see which the best are. For this, we use the objective function.

This function measures the performance of each individual. To be able to judge the quality of an individual and thus compare him to others. The objective function of our case is to minimize the cost while maximizing reliability.

The evaluation of each individual in the population then makes it possible to make the selection. For a system made up of $\mathrm{n}$ components in parallel, the reliability to be maximized is given by equation 2 .

$$
R=1-\prod_{i=1}^{n}\left(1-r_{i}\right)
$$

Where:

- $\quad n$ is the number of components

- $r_{i}$ is the reliability of the component $i$

For a parallel-series system, the reliability to be maximized is given by equation 3 .

$$
R=\prod_{i=1}^{p}\left[1-\prod_{j=1}^{n_{i}}\left(1-r_{i j}\right)\right]
$$

Where:

- $\quad p$ is the number of stages of the system

- $r_{i j}$ the reliability of the $j^{\text {th }}$ element of the $i^{\text {th }}$ stage

- $\quad n_{i}$ is the number of components of the $i^{\text {th }}$ stage

The cost function to be minimized deducted from the work of (Gutha and Vadlamani [9]) for a parallel -series system (machine) with $p$ stages is gave by equation 4.

$$
C=\sum_{i=1}^{p} C_{i}\left[X_{i}+e^{X_{i} / 4}\right]
$$

Where:

- $C_{i}$ is the cost vector of the components of the $X_{i}$ chromosome

- $\quad p$ is the number of stages of the machine.

iv. Selection of the most suitable individuals

When the entire population is assessed at generation $t$, individuals are ranked in ascending order of objective function. Then the selection is made. Selection helps to statistically identify the best individuals in a population and eliminate the bad ones from one generation to the next. This operator also gives a chance to the bad elements because these elements can, by crossing or mutation, generate relevant descendants compared to the optimization criterion.

The first $N \times G$ individuals (the best $N \times G$ ) are selected to be parents. $G$ is the third setting parameter of the genetic method. $G$ is called the generation gap. $G$ makes it possible to select a part of the population to provide sufficient genetic material without decreasing the speed of convergence (Goldberg [6]).

There are different selection techniques:

- Selection by rank: This selection method always chooses the individuals with the best adaptation scores, without allowing chance to intervene;

- Selection by wheel: For each parent, the probability of being selected is proportional to their adaptation to the problem (their score by the fitness function). This selection be imaged by a casino roulette wheel, on which all the chromosomes of the population are placed, the place is given to each of the chromosomes being proportional to its adaptation value. Also, the higher an individual's score, the more likely he is to be selected. We spin the wheel as many times as we want individual sons. The best will be able to be drawn several times, and the worst never;

- Selection by tournament: Two individuals are chosen at random, their adaptation functions are compared, and the best suited is selected.;

- Uniform selection: We are not interested in the adaptation value of the objective function, and the selection is made in a random and uniform manner 
such that each individual has the same probability $P(i)=1 / N$ as all other individuals, where $N$ is the total number of individuals in the population;

- Elitism: The passage from one generation to another through the crossing and mutation operators creates a great risk of losing the best chromosomes. Therefore, elitism aims to copy the best (or first - best) chromosome (s) from the current population to the new population before proceeding to the mechanisms of crossing and mutation. This technique quickly improves the solution because it prevents the loss of the most qualified chromosome when passing from one generation to another.

\section{v. Crossing}

The selected population is divided into $N / 2$ couples formed randomly. Two parents, and are chosen randomly from the potential parents $P_{1}$ and $P_{1}$ their genes are combined according to equation 5 .

$$
a_{j}(k)=a_{j}\left(P_{1}\right)+\left(a_{j}\left(P_{2}\right)-a_{j}\left(P_{1}\right)\right) \times \gamma_{j}
$$

Where:

- $\quad \gamma_{j}$ is a uniform random number,

- $k=N \times G+1, \ldots, N$, the $k$ th individual, mode can

- $j=1, \ldots, m$.

The newly created individual is then evaluated. If its adaptation is better than that of the worst parent, it is integrated into the population to training the next generation. If it is not the case, we repeat the combination.

\section{vi. Mutation of all individuals in the population}

The mutation operator is a process where a minor change in the genetic code is applied to an individual to introduce diversity and thus avoid falling into local optima. This operator is applied with a probability $P_{\mathrm{m}} . P_{\mathrm{m}}$ generally lower than that of the crossing $P_{\mathrm{c}}$. This probability must be low. Otherwise the GA will turn into a random search.

\section{vii. Choosing the best solutions}

This choice consists in retaining the solutions which have a lower value of the objective function, and putting them in the population $\mathrm{P}_{\mathrm{k}+1}$.

\section{viii. Stopping criterion}

The stopping criterion is evaluated in the current population. If it is filled, the whole population has converged on the solution. Otherwise the reproduction pattern will be repeated. The stopping criterion used in this method expresses that all individuals have converged on the same solution and assumes that evolution is no longer possible, that is to say, that no better solution can be found.

The whole strategy is elitist because only the best individuals are selected for survival from one generation to the next and can be the parents of new and better individuals. To ensure convergence of the algorithm, the parameters $N$ and $G$ must be adjusted with care. The size of the population $N$ affects both the performance and efficiency of the algorithm (Bicking et al. [10]). The algorithm is less efficient with very small population sizes. Large population size may contain more interesting solutions and discourage premature convergence towards sub-optimal solutions, but requires more assessments per generation, which can lead to a low convergence rate. The generation gap $G$ determines the proportion of the population that remains unchanged between two generations.

It is chosen to select individuals as severely as possible, without destroying the diversity of the population too much. The global strategy used assumes that all the individuals who make up the population, from generation to generation, satisfy all the constraints.

The best solution for the latest generation represents the solution to the problem by the defined criteria.

\section{ili. Application}

Consider a machine made up of five components mounted in parallel (see Figure 1). Our goal is to define an optimal reliability network, to ensure operation with minimum reliability $r_{\min }=0.80$ while minimizing the cost of the structure.

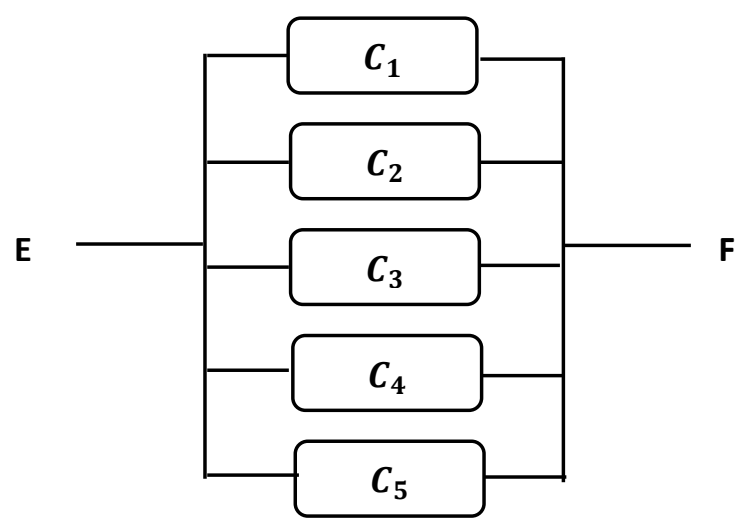

Figure 1: Structure (machine) to optimize

Table 1 groups the parameters of the five components $C_{1}, C_{2}, C_{3}, C_{4}$ and $C_{5}$ :

Table 1: Parameters of the structure components

\begin{tabular}{ccc}
\hline Components & Reliability & Cost \\
\hline $\boldsymbol{C}_{\boldsymbol{1}}$ & 0.95 & 15 \\
\hline $\boldsymbol{C}_{\boldsymbol{2}}$ & 0.97 & 17 \\
\hline $\boldsymbol{C}_{\boldsymbol{3}}$ & 0.9 & 14 \\
\hline $\boldsymbol{C}_{\boldsymbol{4}}$ & 0.89 & 12 \\
\hline $\boldsymbol{C}_{\boldsymbol{5}}$ & 0.96 & 16 \\
\hline
\end{tabular}

Step 1: Data coding

We choose binary coding using a 5-bit character string. Bits 1 through 5 represent the components of $C_{1}$ through $C_{5}$, respectively. If a component exists in the generated solution, then its 
corresponding bit takes the value "1"; otherwise, it takes the value "0".

\section{Step 2: Choice of GA parameters}

This choice is random while waiting to improve it afterward. The parameters of the GA are as follows:

- The size of the population: $N=4$;

- The number of generations: $K=10$;

- The probability of crossing: $P_{c}=0.6$;

- The probability of mutation: $P_{m}=0.01$.

Step 3: Generation of the initial population

We randomly generate a population of 4 noted chromosomes $\mathrm{X}_{1}, \mathrm{X}_{2}, \mathrm{X}_{3}$ and $\mathrm{X}_{4}$ :

$X_{1}=[01001], \quad X_{2}=[10110], \quad X_{3}=[10011]$ and $X_{4}=[11001]$.

Figure 2 represents the reliability network of the configuration corresponding to the chromosome $X_{1}$.

E

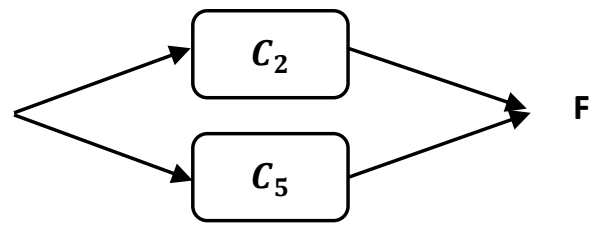

Figure 2: The reliability network corresponding to the chromosome $\mathrm{X}_{1}$.

Step 4: Definition of objective function equation 6

The objective function $f$ to optimize is defined by

$$
\begin{array}{cc}
\text { minimize } & f\left(X_{i}\right)=C\left(X_{i}\right) \\
\text { s.t } & r\left(X_{i}\right) \geq r_{\text {min }}
\end{array}
$$

With:

- $r_{\min }$ : the minimum reliability of the structure to be optimized;

- $\quad C\left(X_{i}\right)$ : the cost of the solution.

Step 5: Evaluation of the reliability of each $X_{i}$ chromosome in the population

For each $X_{i}$ chromosome generated, the reliability and the cost are evaluated respectively by relations 2 and 4 .

So:

$$
\begin{array}{ll}
- & r\left(X_{1}\right)=0.9988 \text { and } f\left(X_{1}\right)=41.25 ; \\
- & r\left(X_{2}\right)=0.99945 \text { and } f\left(X_{2}\right)=66.25 ; \\
- & r\left(X_{3}\right)=0.99978 \text { and } f\left(X_{3}\right)=66.5 ; \\
- & r\left(X_{4}\right)=0.99994 \text { and } f\left(X_{4}\right)=75 ;
\end{array}
$$

Step 6: Selection of the most suitable chromosomes

The best chromosomes in terms of cost in descending order are: $X_{1}, X_{2}, X_{3}$ and $X_{4}$.

We select the chromosomes $X_{1}, X_{2}$ and $X_{3}$ for reproduction and we eliminate the chromosome $X_{4}$ because it is the worst as far as cost is considered.

\section{Step 7: Crossing}

We cross a couple among the selected chromosomes with a crossover rate of 0.6 to form new children. Consider the couple $\left(X_{1}, X_{2}\right)$ :

$$
\left.\begin{array}{l}
X_{1}=[01001] \\
X_{2}=[10110]
\end{array}\right\} \Rightarrow\left\{\begin{array}{l}
X_{1}^{\prime}=[01010] \\
X_{2}^{\prime}=[10101]
\end{array}\right.
$$

Step 8: Mutation

We do a random draw of a single chromosome gene $X_{1}^{\prime}, X_{2}^{\prime}$ and $X_{3}$; then the selected bit will be mutated with a mutation probability of 0.01 . Consider the second bit of the chromosome $\mathrm{X}_{2}^{\prime}$ :

$$
X_{2}^{\prime}=[10101] \Rightarrow X_{2}^{\prime \prime}=[11101]
$$

Step 9: Substitution

We are replacing the new population with the new chromosomes. Chromosomes $X_{3}$ and $X_{4}$ will is eliminated because they are the least suitable, and the chromosome $X_{1}$ and $X_{2}$ will be kept. After all, they are the best qualified among the individuals of the population. So the new population will be made up of chromosomes $X_{1}, X_{2}, X_{1}^{\prime}$ and $X_{2}^{\prime \prime}$.

$X_{1}=[01001], \quad X_{2}=[10110], \quad X_{1}=[01010]$ and $X_{2}^{\prime \prime}=[11101]$.

Step 10: Repeat steps 4 to 9

The algorithm stops either after the reproduction of 16 generations or when we notice that the solution does not improve after a defined number of generations.

Step 11: Optimal solutions

- The corresponding chromosome that optimize objective function is:

$$
\begin{aligned}
& X_{a}=[10001], X_{b}=[11000], X_{c}=[10100], \\
& X_{d}=[01001], X_{e}=[00101], X_{f}=[01010]
\end{aligned}
$$

- The optimum reliability and costs for each solution are:

$$
\begin{aligned}
& r\left(X_{a}\right)=0.998 \text { and } f\left(X_{a}\right)=39, \\
& r\left(X_{b}\right)=0.9985 \text { and } f\left(X_{b}\right)=40.01, \\
& r\left(X_{c}\right)=0.995 \text { and } f\left(X_{c}\right)=40.5, \\
& r\left(X_{d}\right)=0.9986 \text { and } f\left(X_{d}\right)=40.25, \\
& r\left(X_{e}\right)=0.996 \text { and } f\left(X_{e}\right)=37.5, \\
& r\left(X_{f}\right)=0.9967 \text { and } f\left(X_{f}\right)=36.25, \\
& r\left(X_{g}\right)=0.989 \text { and } f\left(X_{g}\right)=32.5 .
\end{aligned}
$$

By considering the components selected by the genetic algorithm in the previous optimal solutions, we generate all the possible paths (connections) between the component of the machine. In solution $1\left(X_{a}\right.$ chromosome), we retain the components in parallel $\mathrm{C}_{1}$ 
and $\mathrm{C}_{5}$; in solution 2 , we retain the components $\mathrm{C}_{1}$ and $\mathrm{C}_{2}$ (thus defining a path); in solution 3 , we retain the components $\mathrm{C}_{1}$ and $\mathrm{C}_{3}$ (defining a path) and from end to end until the last solution ( $\mathrm{X}_{\mathrm{g}}$ chromosome), the last path connecting the components $\mathrm{C}_{3}$ and $\mathrm{C}_{4}$ is shown. From where the network of optimal reliability of the machine is given by Figure 3.

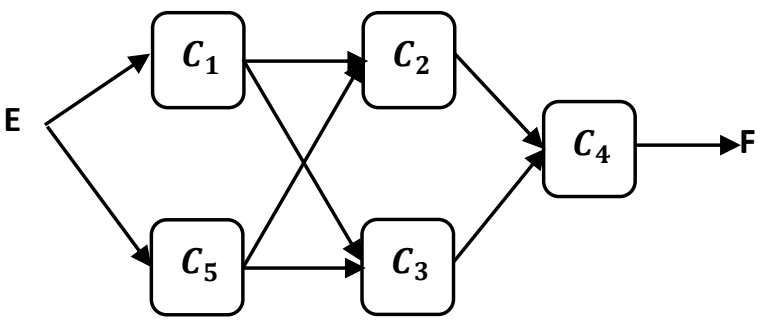

Figure 3: Optimal network reliability of our machine

By evaluating the overall reliability of the reliability network, we obtain a reliability of 0.88 , higher than the minimum reliability set. Similarly, by evaluating the overall cost of the machine by applying relation 4 , for the reliability network we obtain a cost of 88.75 , which is much lower than the overall cost generated by the initial system, which is 103.75. Objectively, the reliability network has favored cost reduction on the one hand reliability increase on the other.

\section{Conclusion}

Having completed the writing of this paper, which concerns the optimization of the reliability of a system by the genetic algorithm, it appears that the general objective has been achieved. Indeed, through the functionality of genetic algorithms, implemented on a structure with five components, we were able to reduce the cost and increase the reliability of the parallel structure. However, although the genetic algorithm is easy to implement, it might require an infinite number of iterations to the best approach of optimal solution. This would make the algorithm less robust. In perspective for this work, it would be wise to develop or implement another meta-heuristic that would not require a large number of iterations.

Data Availability

The data needed to support these results are available in the text, specifically in Table 1. Conflicts of Interest All authors declare that there are no conflicts of interest regarding the publication of this paper.

\section{ACKNOWLEDGMENTS}

This work was funded by the authors of the paper and was carried out in the Department of Fundamental Sciences and Engineering Techniques at the Chemical Engineering and Mineral Industries School at the University of Ngaoundere.

\section{References Références Referencias}

1. L. Painton and J. Campbell, "Genetic algorithm in optimization of system reliability", IEEE Trans Reliab, 44:172-8, 1995.

2. G. Levitin and A. Lisnianski, "Optimizing survivability of vulnerable series-parallel multi-state systems", Reliability Engineering and System Safety, 79:319331, 2003.

3. I. Reichenberg, "Cybernetic solution path of an experimental poblem", Library Translation 1122, Royal Aircraft Establishment, Famborough, UK, 1965.

4. J. H. Holland, "Adaptation in natural and artificial systems", University of Michigan press, 1975.

5. D. Goldberg, "Genetic algorithms", Addison Wesley, ISBN, 0- 201-15767-5, 1989a.

6. D. Goldberg, "Genetic algorithms in search, optimization and machine learning", Addison Wesley, 1989b.

7. S.M. Douiri, S. Elbarnoussi and H. Lakhbab, "Course of Heuristic and Metaheuristic Exact Resolution Methods', Mohammed V University, Faculty of Sciences of Rabat, Morocco, Mathematics, Computer Science and Applications Research Laboratory, 2013.

8. H.P. Schewefel, "Numerical Optimization of computer models, Editions Wiley, 1981.

9. J. K Gutha, and R. Vadlamani, "Modified Harmony Search Applied to Reliability Optimization of Complex Systems", Advances in Intelligent Systems and Computing 382, 2016.

10. F. Bicking, C. Fonteix, J.P. Corriou, and I. Marc, "Global optimization by artificial life: a new technique using genetic population evolution", RAIRO-Operations Research, vol. 28(1), 23-36, 1994. 